\title{
Identifying Chinese Herbal Medicine Network for Eczema: Implications from a Nationwide Prescription Database
}

\author{
Hsing-Yu Chen, ${ }^{1,2,3}$ Yi-Hsuan Lin, ${ }^{1,2,3,4}$ Sindy Hu, ${ }^{2,4,5}$ Sien-hung Yang, ${ }^{1,3}$ \\ Jiun-liang Chen, ${ }^{1,3}$ and Yu-Chun Chen ${ }^{6,7}$ \\ ${ }^{1}$ Division of Chinese Internal Medicine, Center for Traditional Chinese Medicine, Chang Gung Memorial Hospital, \\ Taoyuan 33378, Taiwan \\ ${ }^{2}$ Graduate Institute of Clinical Medical Sciences, College of Medicine, Chang Gung University, Taoyuan 33302, Taiwan \\ ${ }^{3}$ School of Traditional Chinese Medicine, College of Medicine, Chang Gung University, Taoyuan 33302, Taiwan \\ ${ }^{4}$ Department of Cosmetic Science, Chang Gung University of Science and Technology, Taoyuan 33302, Taiwan \\ ${ }^{5}$ Department of Dermatology, Chang Gung Memorial Hospital, Taoyuan 33378, Taiwan \\ ${ }^{6}$ Department of Medical Research and Education, National Yang-Ming University Hospital, Ilan 26042, Taiwan \\ ${ }^{7}$ Institute of Hospital and Health Care Administration, School of Medicine, National Yang-Ming University, Taipei 112, Taiwan
}

Correspondence should be addressed to Yu-Chun Chen; yuchn.chen@googlemail.com

Received 8 July 2014; Revised 3 October 2014; Accepted 4 October 2014

Academic Editor: Karl Wah-Keung Tsim

Copyright (C) 2015 Hsing-Yu Chen et al. This is an open access article distributed under the Creative Commons Attribution License, which permits unrestricted use, distribution, and reproduction in any medium, provided the original work is properly cited.

\begin{abstract}
Eczema is a highly prevalent dermatological disease that can severely affect the patient's quality of life. Chinese herbal medicine $(\mathrm{CHM})$ is commonly used in combination for eczema due to the complicated pathogenesis. This study aimed to identify a CHM network for the treatment of eczema by using a nationwide database. During 2011, 381,282 CHM prescriptions made for eczema (ICD-9-CM 692.x) were obtained from the National Health Insurance Research Database (NHIRD) in Taiwan and analyzed by using association rule mining and social network analysis. Among 661 available CHMs, 44 important combinations were identified. Among the CHM networks, seven clusters with the predominant traditional Chinese medicine (TCM) pattern were recognized. The largest CHM cluster was used to treat the wind-dampness-heat pattern, and Xiao-Feng-San (24.1\% of all prescriptions) was the core of this cluster with anti-inflammation, antioxidation, and antiallergic effects. Lonicera japonica (11.0\% of all prescriptions) with Forsythia suspense (17.0\% of all prescriptions) was the most commonly used CHM combination and was also the core treatment for treating the heat pattern, in which an antimicrobial effect is found. CHM network analysis is helpful for TCM doctors or researchers to choose candidates for clinical practice or further studies.
\end{abstract}

\section{Introduction}

Eczema is a prevalent chronic inflammatory dermatological disease, with an increasing prevalence and financial burden, especially in Asian countries [1, 2]. In addition to being highly associated with other comorbidities, eczema is characterized by frequent relapses of skin itching, erythema, scratching, and discharge and thus can severely affect the patients' quality of life $[1,3]$. While there is no definite cure for eczema, antihistamines, corticosteroids, emollients, and even immune-modulating agents are often used concurrently to control symptoms. Corticosteroids are one of the most important and effective treatments for eczema caused by a Th2 dominant immune response $[4,5]$. However, recurrent symptoms of eczema commonly lead to the prolonged use of corticosteroids which can cause side effects, including skin atrophy and angioectasia that can easily lead to bleeding, immunosuppression, euphoria, and endocrine dysfunction $[6,7]$. Due to the inadequacy of current treatment, finding an optimal treatment strategy to treat eczema without longterm complications remains an urgent issue $[4,8]$.

Traditional Chinese medicine (TCM) is one of the most commonly used alternative medicines in Taiwan due to the perceived favorable efficacy with fewer side effects $[9,10]$. 
Increases in the cost and use of TCM including Chinese herbal medicine (CHM), acupuncture, and massage have been reported in recent years. With regard to treatment for eczema, CHM is much more commonly used than other modalities of TCM [11]. Despite the high prevalence of the use of CHM, the composition of CHM prescriptions for eczema is unclear and such investigations are lacking. Analyzing these prescriptions is important to clarify the role CHM may play in the treatment of eczema and also in the selection of suitable candidates for clinical trials and bench studies, especially when the CHM used in daily practice may be different from classic TCM $[12,13]$. In addition, the high heterogeneity in $\mathrm{CHM}$ reported in meta-analyses of systematic reviews may be related to a lack of information on the CHM prescriptions used in clinical practice $[11,13]$.

A CHM network is helpful in understanding the rationale of CHM prescriptions through a graphical demonstration of relationships between CHMs [14]. Network analysis is especially important when trying to understand how prescriptions are made, since TCM doctors frequently combine five to six CHMs in one prescription from hundreds of available CHMs $[15,16]$. The choice of the CHMs is mainly dependent on the characteristics of the other CHMs, such as four qi and five flavors, in the same prescription. Specific connections between pairs of CHMs, such as herbal pairs, are the key components of CHM prescriptions, and thus a sophisticated CHM network can be formed [14, 17]. In addition, further studies on the efficacy and active ingredients may be facilitated on the basis of a CHM network [18-20].

This study aimed to identify a CHM network for the treatment of eczema, composed of commonly used CHMs and combinations by analyzing a nationwide prescription database. These findings are not only helpful in knowing the rationale of CHM formulation but also beneficial to further studies when selecting their candidates.

\section{Material and Methods}

2.1. Data Source. A cross-sectional CHM prescription dataset obtained from the National Health Insurance Research Database (NHIRD), Taiwan, was used in this study. The National Health Insurance (NHI) program was established in Taiwan in 1995 and currently provides comprehensive medical care for over $99 \%$ of the 23 -million population. All information required for NHI reimbursement, including gender, birth date, reasons for visits and interventions (including medication, frequency, and duration), examinations ordered by the doctors, prescriptions, and expenses, is digitized and stored in the NHIRD. The patient's identification number is encrypted in the NHIRD, and thus an individual's true identity cannot be traced. To verify the reason for each visit, up to three diagnoses are recorded using International Classification of Diseases, Ninth Revision, Clinical Modification (ICD-9-CM) codes. Furthermore, the first diagnosis code is required to be the main reason for each visit. The reliability of ICD-9-CM coding as reasons for visits has been proven, and the NHIRD itself has been successfully used for many studies including core treatments from prescription analysis and analysis of the characteristics of patients receiving TCM
$[10,21,22]$. In addition, the utilization of CHM is unique in Taiwan. Reimbursements for both CHM and western medicine (WM) are made equally, and therefore patients are free to choose treatments without bias. Due to the high coverage of the NHI, the results of analysis using prescriptions can be seen as a general consensus, and potential selection or referral bias can therefore be effectively eliminated $[16,22]$.

2.2. Study Subjects. To identify visits made for TCM treatments for eczema, a single diagnosis of eczema (ICD-9CM code: 692.x) was used. Eczema consists of a range of persistent dermatologic manifestations, including skin pruritus, erythema, swelling, and lichenification with prominent scratching. Visits with a first ICD-9-CM code other than 692.x or other ICD-9-CM codes in the second or third diagnosis were excluded to minimize potential confounding bias on the prescription caused by comorbidities.

2.3. Chinese Herbal Medicine Prescription Dataset. All visits with CHM prescriptions for eczema during 2011 were used to construct the CHM prescription dataset. Herbal formulas ( $\mathrm{HF}$ ) and single herbs $(\mathrm{SH})$ are the only kinds of CHM reimbursed by the NHI in Taiwan. SH, such as Angelica sinensis (Dang Gui), include herbs, animal parts, and minerals recorded in TCM materia medica while HF, such as Xiao-Feng-San (XFS), are composed of several $\mathrm{SH}$ with fixed proportions according to TCM classics. HF and $\mathrm{SH}$ are processed into a concentrated powder form, and TCM doctors are able to freely combine multiple $\mathrm{HF}$ or $\mathrm{SH}$ into one prescription to achieve the therapeutic goals.

2.4. Statistical Analysis. Applying association rule mining (ARM) with social network analysis (SNA) on large-scale prescription datasets is helpful in exploring CHM networks, as we previously reported $[15,16]$. ARM is used to identify commonly used CHMs and their combinations, while the CHM network can be established by incorporating CHM combinations in SNA. ARM is one of the most commonly used data-mining techniques and has been used extensively to explore relationships between study targets such as combinations of CHMs, coprescriptions of WM drugs, comorbidities of diseases, and TCM syndrome [23-27]. Two decisive factors, support factor and confidence factor, are used in the ARM model to explore commonly used CHMs and CHM combinations. Support factor represents the prevalence of an individual CHM, and confidence factor represents the strength of connection between CHMs. A higher support factor of a CHM means that the CHM is used more commonly, while a higher level of confidence factor between two CHMs means the relationship is closer. The detailed algorithm was described in our previous work [28]. The thresholds of support and confidence factors were set to $1 \%$ and $30 \%$, respectively, according to our experience in the analysis of CHM for urticaria [15].

The relationships between CHMs are illustrated by SNA, which is a powerful analytic method to present a sophisticated CHM network by using the hierarchical cluster method $[16,28,29]$. Several measurements are used to characterize 
TABLE 1: Age and gender distribution of Chinese herbal medicine (CHM) users $(N=132,971)$.

\begin{tabular}{lc}
\hline & $\begin{array}{c}\text { CHM users } \\
n(\%)\end{array}$ \\
\hline $\begin{array}{l}\text { Gender } \\
\text { Female }\end{array}$ & \\
Male & $83241(62.6)$ \\
Age (years) & $49730(37.4)$ \\
$0-10$ & \\
$11-20$ & $7092(5.3)$ \\
$21-30$ & $27128(20.4)$ \\
$31-40$ & $32981(24.8)$ \\
$41-50$ & $25905(19.5)$ \\
$51-60$ & $18741(14.1)$ \\
$61-70$ & $11991(9.0)$ \\
$>70$ & $5153(3.9)$ \\
\hline
\end{tabular}

the CHM network, including modularity, degree, and centrality $[30,31]$. Degree represents the frequency of connections to a certain $\mathrm{CHM}$, and modularity represents the fitness of CHM clustering. Once the best modularity has been acquired, CHMs with closer relationships are classified as one cluster with relationships of different clusters being as distant as possible. The detailed algorithm was reported in a previous study [30]. This graphical presentation of CHM combination patterns is helpful to understand the role of CHM in treating various diseases, including eczema. The open-source software " $\mathrm{R}$ " with the "arule" package was used to obtain important CHM combinations and visualize the CHM network in this study.

\section{Results}

There were 381,282 CHM prescriptions made for 132,971 eczema patients in 2011. Most of the CHM users were female (62.6\%), most were aged between 21 and 30, and more than two-thirds were adults (Table 1). A total of $661 \mathrm{CHMs}$, including $\mathrm{SH}$ and HF, were used, and an average of approximately 5.8 CHMs was used per prescription on average. Multiple CHMs were commonly used in one prescription, and more than $90 \%$ of the prescriptions were composed of at least two CHMs (Figure 1).

Of the 661 available CHMs used to treat eczema in the CHM prescription dataset, the top 10 most commonly used CHMs (including $\mathrm{SH}$ and HF) are shown in Tables 2 and 3 . XFS was the most commonly used HF (24.1\%), followed by Qing-Shang-Fang-Feng-Tang (14.9\%), Xian-Fang-HuoMing-Yin (12.5\%), and Huang-Lian-Jie-Du-Tang (10.9\%) (Table 2). Among all SH, Forsythia suspensa (Lian Qiao) was most commonly used (17.0\%), followed by Coix lacrymajobi (Yi Yi Ren) and Taraxacum mongolicum (Pu Gong Ying), accounting for $12.7 \%$ and $12.1 \%$ of all prescriptions, respectively (Table 3 ). The TCM doctors usually prescribed $\mathrm{HF}$ at three to four times higher dosage than $\mathrm{SH}$, at about $4 \mathrm{gm} /$ day for HF and 1-1.5 gm/day for SH (Tables 2 and 3).

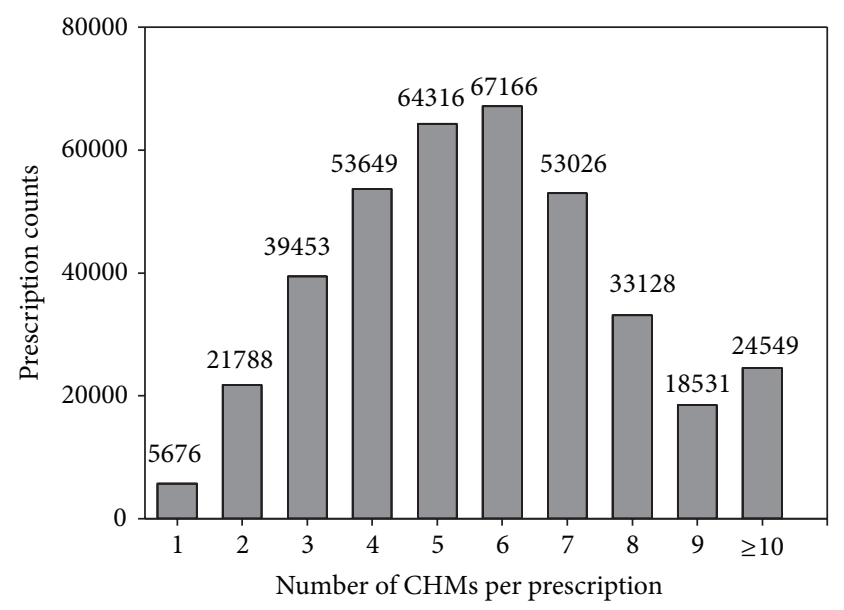

FIGURE 1: Distribution of the number of prescriptions.

Forty-four important CHM combinations were investigated in this study, the top 10 most common of which are listed in Table 4. Lonicera japonica (Jin Yin Hua) with Forsythia suspensa (Lian Qiao) was the most common combination (5.0\% of all prescriptions), followed closely by XFS with Dictamnus dasycarpus (Bai Xian Pi) (4.8\% of all prescriptions) (Table 4). XFS seemed to have an important role in treating eczema since it was used in 4 out of 10 combinations. In addition, the importance of its role in treating eczema was clearly shown when all significant combinations of $\mathrm{CHMs}$ were clustered by SNA.

Seven clusters were found in the CHM network for treating eczema by analyzing all $\mathrm{CHM}$ combinations as illustrated in Figure 2, in which larger circles and thicker connection lines represented a higher prevalence of the $\mathrm{CHM}$ and the combination, respectively. Each cluster had a predominant TCM syndrome as indicated when applying the characteristics of each CHM to the network; for example, the $\mathrm{CHMs}$ for wind dampness and heat syndrome were predominantly grouped in cluster 1 (Figure 2). CHMs in the same cluster had strong interconnections, while some clusters were used in combination, for example, cluster 1 (winddampness-heat syndrome) with cluster 6 (wind syndrome) and cluster 2 (heat-toxin syndrome) with clusters 4 (blood heat), 5 (heat toxin), or 6 (wind). XFS was at the center of the biggest cluster in the CHM network (Figure 2).

The pharmacological mechanisms of CHM were then accessed in a PubMed search and are summarized in Table 5 (last accessed date: 2014/6/30). Antioxidation, antiinflammation, and antiallergic effects were frequently found among the commonly used CHMs. However, XFS was the only CHM to reportedly have all the effects. Interestingly, antimicrobial effects were reported in some of the CHMs, including Huang-Lian-Jie-Du-Tang, Forsythia suspensa (Lian Qiao), and Lonicera japonica (Jin Yin Hua).

\section{Discussion}

To the best of our knowledge, this is the first nationwide study to investigate a CHM network for the treatment of eczema. 
TABLE 2: The top 5 most commonly prescribed herbal formulas for eczema during 2011 (total prescriptions $=381,282$ ).

\begin{tabular}{|c|c|c|c|c|}
\hline Herbal formulas & Ingredients (English name) & $\begin{array}{l}\text { Dosage } \\
\text { (gm/day) }\end{array}$ & TCM indications & Number of prescriptions (\%) \\
\hline $\begin{array}{l}\text { Xiao-Feng-San } \\
\text { (XFS) }\end{array}$ & $\begin{array}{l}\text { Saposhnikovia divaricata (Fang Feng), } \\
\text { Atractylodes lancea (Bai Zhu), } \\
\text { Schizonepeta tenuifolia (Jing Jie), } \\
\text { Arctium lappa (Niu Bang Zi), } \\
\text { Glycyrrhiza uralensis (Gan Cao), } \\
\text { Rehmannia glutinosa (Sheng Di Huang), } \\
\text { Gypsum Fibrosum (Shi Gao), } \\
\text { Caulis clematidis armandii, } \\
\text { Anemarrhena asphodeloides (Mu Tong), } \\
\text { Angelica sinensis (Dang Gui), } \\
\text { Cryptotympana pustulata (Chan Tui), } \\
\text { Sesamum indicum (Hei Zhi Ma), } \\
\text { Sophora flavescens (Ku Shen). }\end{array}$ & 4.77 & $\begin{array}{c}\text { Wind and } \\
\text { dampness heat }\end{array}$ & $92075(24.1)$ \\
\hline $\begin{array}{l}\text { Qing-Shang-Fang- } \\
\text { Feng-Tang }\end{array}$ & $\begin{array}{l}\text { Saposhnikovia divaricata (Fang Feng), } \\
\text { Coptis chinensis (Huang Lian), } \\
\text { Ligusticum chuanxiong (Chuan Xiong), } \\
\text { Platycodon grandiflorum (Jie Geng), } \\
\text { Forsythia suspensa (Lian Qiao), } \\
\text { Scutellaria baicalensis (Huang Qin), } \\
\text { Mentha haplocalyx (Bo He), } \\
\text { Angelica dahurica (Bai Zhi), } \\
\text { Schizonepeta tenuifolia (Jing Jie), } \\
\text { Glycyrrhiza uralensis (Gan Cao), } \\
\text { Citrus aurantium (Zhi Qiao). }\end{array}$ & 4.67 & $\begin{array}{l}\text { Wind heat and } \\
\text { toxin }\end{array}$ & 56903 (14.9) \\
\hline $\begin{array}{l}\text { Xian-Fang-Huo- } \\
\text { Ming-Yin }\end{array}$ & $\begin{array}{l}\text { Lonicera japonica (Jin Yin Hua), } \\
\text { Saposhnikovia divaricata (Fang Feng), } \\
\text { Angelica dahurica (Bai Zhi), } \\
\text { Angelica sinensis (Dang Gui), } \\
\text { Paeonia lactiflora (Shao Yao), } \\
\text { Commiphora myrrha (Mo Yao), } \\
\text { Fritillaria thunbergii (Zhe Bai Mu), } \\
\text { Trichosanthes kirilowii (Tian Hua Fen), } \\
\text { Gleditsia sinensis (Zao Jiao), } \\
\text { Citrus reticulate (Chen Pi), } \\
\text { Glycyrrhiza uralensis (Gan Cao). }\end{array}$ & 4.21 & Heat toxin & $47521(12.5)$ \\
\hline $\begin{array}{l}\text { Huang-Lian-Jie- } \\
\text { Du-Tang }\end{array}$ & $\begin{array}{l}\text { Gardenia jasminoides (Zhi Zi), } \\
\text { Scutellaria baicalensis (Huang Qin), } \\
\text { Coptis chinensis (Huang Lian), } \\
\text { Phellodendron chinense (Huang Bai) }\end{array}$ & 3.91 & Heat toxin & 41451 (10.9) \\
\hline $\begin{array}{l}\text { Jia-Wei-Xiao-Yao- } \\
\text { San }\end{array}$ & $\begin{array}{l}\text { Paeonia lactiflora (Shao Yao), } \\
\text { Bupleurum chinense (Chai Hu), } \\
\text { Atractylodes macrocephala (Bai Zhu), } \\
\text { Poria cocos (Fu Ling), } \\
\text { Angelica sinensis (Dang Gui), } \\
\text { Mentha haplocalyx (Bo He), } \\
\text { Glycyrrhiza uralensis (Gan Cao), } \\
\text { Zingiber officinale (Gan Jiang), } \\
\text { Paeonia suffruticosa (Mu Dan Pi), } \\
\text { Gardenia jasminoides (Zhi Zi). }\end{array}$ & 4.13 & $\begin{array}{l}\text { Liver qi stagnation } \\
\text { with heat, spleen qi } \\
\text { deficiency }\end{array}$ & $36423(9.6)$ \\
\hline
\end{tabular}

In this study, most CHM users were adult females, which is compatible with previous reports in which the peak age of the occurrence of eczema was reported to be from 18 to 29 years $[50,51]$. The TCM doctors commonly prescribed multiple CHMs with an average of 5.8 CHMs per prescription and more than $90 \%$ of the prescriptions containing more than 3 CHMs in this study (Figure 1). This result is similar to our previous work on urticaria (an average of 5.46 CHMs per prescription), and this may be related to the fact that both urticaria and eczema are diseases with a complicated pathogenesis [15].

We propose a CHM network to treat eczema including the commonly used CHMs and their relationships in this study, and this network may be beneficial to understand 
TABle 3: The top 10 most commonly used single herbs for eczema during 2011 (total prescriptions $=381,282$ ).

\begin{tabular}{|c|c|c|c|c|}
\hline $\begin{array}{l}\text { Single herb } \\
\text { Latin name }\end{array}$ & English name & Dosage (gm/day) & TCM indications & Number of prescriptions (\%) \\
\hline Forsythia suspensa & Lian Qiao & 1.20 & Heat toxin & $64868(17.0)$ \\
\hline Coix lacryma-jobi & Yi Yi Ren & 1.34 & $\begin{array}{l}\text { Dampness, spleen qi } \\
\text { deficiency, and heat }\end{array}$ & $48581(12.7)$ \\
\hline Taraxacum mongolicum & Pu Gong Ying & 1.30 & Heat & $16101(12.1)$ \\
\hline Dictamnus dasycarpus & Bai Xian Pi & 1.26 & Dampness and heat & $41897(11.0)$ \\
\hline Lonicera japonica & Jin Yin Hua & 1.21 & Heat toxin & $41895(11.0)$ \\
\hline Glycyrrhiza uralensis & Gan Cao & 0.87 & $\begin{array}{c}\text { Spleen qi deficiency, } \\
\text { moderation of } \\
\text { properties of other drugs }\end{array}$ & $39814(10.4)$ \\
\hline Rheum palmatum & Da Huang & 1.34 & $\begin{array}{l}\text { Heat toxin and blood } \\
\text { heat }\end{array}$ & $38194(10.0)$ \\
\hline Paeonia suffruticosa & Mu Dan Pi & 1.38 & Blood heat, stasis & $33929(8.9)$ \\
\hline Kochia scoparia & Di Fu Zi & 1.56 & Heat & $33417(8.8)$ \\
\hline Smilax glabra & Tu Fu Ling & 1.41 & Dampness and heat & $32565(8.5)$ \\
\hline
\end{tabular}

TABLE 4: Top 10 pairs of Chinese herbal medicines (CHMs) used in combination for eczema.

\begin{tabular}{lllcc}
\hline CHM A (English name) & & CHM B (English name) & Instances & Prevalence (\%) \\
\hline Lonicera japonica (Jin Yin Hua) & With & Forsythia suspensa (Lian Qiao) & 18873 & 5.0 \\
Xiao-Feng-San & With & Dictamnus dasycarpus (Bai Xian Pi) & 18293 & 14816 \\
Taraxacum mongolicum (Pu Gong Ying) & With & Forsythia suspensa (Lian Qiao) & 14001 & 3.9 \\
Dictamnus dasycarpus (Bai Xian Pi) & With & Kochia scoparia (Di Fu Zi) & 13993 & 3.7 \\
Xiao-Feng-San & With & Kochia scoparia (Di Fu Zi) & 13390 & 3.7 \\
Coix lacryma-jobi (Yi Yi Ren) & With & Kochia scoparia (Di Fu Zi) & 12776 & 3.5 \\
Qing-Shang-Fang-Feng-Tang & With & Taraxacum mongolicum (Pu Gong Ying) & 3.4 \\
Xiao-Feng-San & With & Forsythia suspensa (Lian Qiao) & 11980 & 3.1 \\
Xiao-Feng-San & With & Coix lacryma-jobi (Yi Yi Ren) & 11900 & 3.1 \\
Xian-Fang-Huo-Ming-Yin & With & Forsythia suspensa (Lian Qiao) & 2.9 \\
\hline
\end{tabular}

the complicated CHM treatment model, "TCM pattern identification and treatment," or "bian-zheng-lun-zhi" in Chinese $[14,19]$. “TCM pattern," or zheng, is a concise summary of the patient's symptoms according to TCM theory, and CHMs are prescribed for the TCM pattern. Due to variety in the patient's manifestations, several TCM patterns may be identified for a single disease, and it may exist to a different extent in each patient. To cope with these combined TCM patterns, TCM doctors usually use several CHMs aimed at certain patterns in one prescription, and thus a CHM pharmacology network is used with the concept of "multiple target, complex diseases" $[14,19]$. Therefore, investigating CHM combinations and the construction of a CHM network is more important than studying only one individual CHM. To achieve this goal, SNA was used to graphically demonstrate the CHM network in this study. Modularity, the decisive parameter used to explore clusters, resulted in each cluster of CHMs having strong within-cluster connections and weak between-cluster connections, and this process is similar when prescribing CHMs. TCM doctors usually choose a group of CHMs for the main TCM pattern and then add other CHMs for minor
TCM patterns or symptoms. Because of this, SNA has been used to analyze CHM networks for many diseases [15, 16, 28].

Our proposed CHM network for eczema is graphically demonstrated in Figure 2 and may be a valuable reference for TCM doctors when choosing CHMs for certain TCM patterns among eczema patients from the enormous number of available CHMs. When applying the characteristics of the CHM into the CHM network, the TCM pattern-CHM relationships could be seen, even though the information on the TCM pattern of each patient was not provided in the original dataset. The CHM for the wind-dampness-heat pattern constituted the largest cluster, while the heat pattern existed in nearly all of the clusters, which is similar to a previous investigation [52]. The dampness-heat pattern is characterized by skin itching, erythema, local heat, discharge, and swelling in relapse and is usually caused by chronic inflammatory dermatological processes $[53,54]$. These manifestations are diagnostic features of eczema, and the high prevalence of this pattern may be related to the wet climate in Taiwan [55]. Moreover, the wind pattern is characterized by rapid movements and swift changes in dermatological 


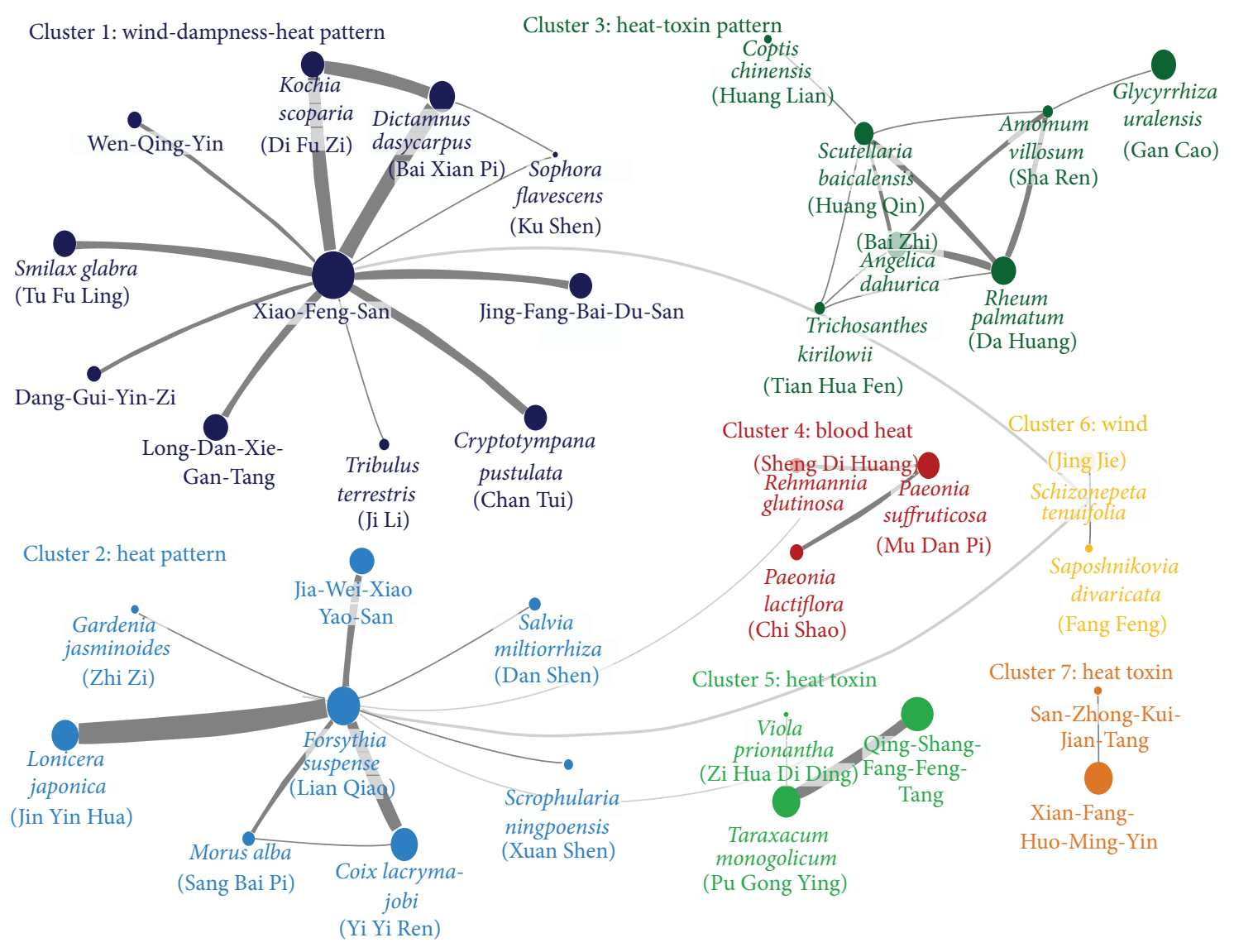

FIGURE 2: Chinese herbal medicine network for eczema.

symptoms and also presents as the migratory skin lesions around the whole body commonly found among eczema patients. Consequently, combinations of wind, dampness, and heat comprise the majority of TCM patterns among eczema patients and therefore specific CHMs are used for the various combinations of these patterns.

XFS was found to be the most important CHM to treat eczema of all the CHMs, since it was the core of cluster 1 (wind-dampness-heat pattern) and many other CHMs were combined with XFS to effectively treat eczema (Table 4). XFS has been used as a CHM for hundreds of years to expel wind dampness and heat for itching, erythema, and swelling skin lesions with discharge, and its efficacy in treating atopic dermatitis has been proven [56]. In addition, XFS has been reported to have more extensive anti-inflammatory, antiallergic, and antioxidative effects in treating eczema compared with other CHMs used for eczema [45-48]. By modulating the imbalance of Th1 and Th2 cells, XFS can decrease levels of serum IL-4 and interferon gamma which may then correct the constitution of the patient and relieve symptoms [48]. This wide range of action on both pharmacological mechanism and TCM pattern may be the reason why XFS is the most important CHM in the treatment of eczema.

The additive effects of other CHMs combined with XFS are also noteworthy. TCM doctors usually use other CHMs with XFS either to enhance the effectiveness on major pattern or to cover other minor TCM patterns, and these combinations of multiple CHMs can be easily seen in the CHM network diagram. The combination of XFS, Kochia scoparia (Di Fu Zi), and Dictamnus dasycarpus (Bai Xian $\mathrm{Pi}$ ) is the major component of cluster 1, and the addition of Kochia scoparia (Di Fu Zi) and Dictamnus dasycarpus (Bai Xian Pi) may enhance the efficacy of XFS on expelling dampness and heat via enhancing the anti-inflammation and antiallergic effects $[39,44,57]$. In addition, Lonicera japonica (Jin Yin Hua) is usually combined with Forsythia suspensa (Lian Qiao) and forms the center of cluster 2 to treat the heat pattern of eczema. This combination is also commonly used with cluster 6, Schizonepeta tenuifolia (Jing Jie) and Saposhnikovia divaricata (Fang Feng), which are well known CHM pair used to expel pathogenic wind. This combination may have combined effects on treating patients with the major heat pattern and minor wind pattern. The unclear pharmacological mechanisms of Schizonepeta tenuifolia (Jing Jie) and Saposhnikovia divaricata (Fang Feng), especially when used in combination, warrant future studies since Lonicera japonica (Jin Yin Hua) and Forsythia suspensa (Lian Qiao) already cover nearly all of the pathogenesis of eczema [33-35, 58, 59].

The antimicrobial effect is another interesting finding in the CHM network, in which nearly all clusters had a CHM with antimicrobial effects. Many CHMs with the ability to 
TABle 5: Possible pharmacological mechanisms of Chinese herbal medicines (CHMs) used for eczema.

\begin{tabular}{|c|c|}
\hline $\mathrm{CHM}$ & Possible mechanisms \\
\hline \multicolumn{2}{|l|}{ Single herb (SH) } \\
\hline Forsythia suspensa (Lian Qiao) & $\begin{array}{l}\text { Antioxidation [32] } \\
\text { Anti-inflammation [33] } \\
\text { Antiallergy effect [34] } \\
\text { Antibacterial effect [35] }\end{array}$ \\
\hline Coix lacryma-jobi (Yi Yi Ren) & $\begin{array}{l}\text { Anti-inflammation/antiallergic } \\
\text { effect [36] }\end{array}$ \\
\hline $\begin{array}{l}\text { Taraxacum mongolicum (Pu } \\
\text { Gong Ying) }\end{array}$ & Antioxidation [37] \\
\hline $\begin{array}{l}\text { Dictamnus dasycarpus (Bai } \\
\text { Xian } \mathrm{Pi} \text { ) }\end{array}$ & $\begin{array}{l}\text { Antiallergic effect [38] } \\
\text { Anti-inflammation [39] }\end{array}$ \\
\hline $\begin{array}{l}\text { Lonicera japonica (Jin Yin } \\
\text { Hua) }\end{array}$ & $\begin{array}{l}\text { Anti-inflammation [40] } \\
\text { Antiallergic effect [41] }\end{array}$ \\
\hline $\begin{array}{l}\text { Glycyrrhiza uralensis (Gan } \\
\text { Cao) }\end{array}$ & $\begin{array}{l}\text { Anti-inflammation/antioxidation } \\
\text { [42] }\end{array}$ \\
\hline Rheum palmatum (Da Huang) & Antiallergic effect [43] \\
\hline $\begin{array}{l}\text { Paeonia suffruticosa (Mu Dan } \\
\mathrm{Pi} \text { ) }\end{array}$ & Antiallergic effect [41] \\
\hline Kochia scoparia (Di Fu Zi) & Antiallergic effect [44] \\
\hline \multicolumn{2}{|l|}{ Herbal formula (HF) } \\
\hline Xiao-Feng-San & $\begin{array}{l}\text { Antiallergic effect }[45,46] \\
\text { Antioxidation/anti-inflammation } \\
\text { [47] } \\
\text { Immunomodulation of Th1/Th2 } \\
\text { balance [48] }\end{array}$ \\
\hline Huang-Lian-Jie-Du-Tang & $\begin{array}{l}\text { Anti-inflammation, decreasing } \\
\text { cellular adhesion molecule } \\
\text { expression [49] } \\
\text { Immunomodulation of Th1/Th2 } \\
\text { balance [48] }\end{array}$ \\
\hline
\end{tabular}

treat the heat pattern have potent antimicrobial effects, such as Huang-Lian-Jie-Du-Tang, Forsythia suspensa (Lian Qiao), Lonicera japonica (Jin Yin Hua), Coix lacryma-jobi (Yi Yi Ren), and Dictamnus dasycarpus (Bai Xian Pi) [35, 60-62]. This finding implies the crucial role of microbial infections in relapsing eczema, which has only been proposed in early reports; however, such infections are not routinely treated due to concerns of drug resistance $[63,64]$. Nonetheless, the use of CHM may enhance innate immunity and provide an alternative method to control microbial infections as well as to directly eradicate microbes. For example, Huang-LiangJie-Du-Tang has been reported to increase the phagocytic ability of macrophages, and Coix lacryma-jobi (Yi Yi Ren) has been reported to increase the number of peripheral cytotoxic T cells and NK cells $[60,62]$. Consequently, combining these antimicrobial CHMs with conventional therapy may be a potential therapy to control refractory eczema.

\section{Conclusion}

This study is the first pharmacoepidemiological study to propose a CHM network for the treatment of eczema. Through visualization of the network, core CHMs and CHM combinations for the corresponding TCM patterns and the rationale behind the $\mathrm{CHM}$ formulations can clearly be seen. These results of this study can be regarded as a consensus among TCM doctors in Taiwan, and they may therefore provide a valuable reference for both TCM doctors and researchers to select suitable candidates for clinical practice or future studies.

\section{Ethical Approval}

The study protocol was approved and certification was exempted by the Institutional Review Board of the Chang Gung Memorial Foundation, Taipei, Taiwan (no. 101-2290b).

\section{Conflict of Interests}

The authors declare that there is no conflict of interests regarding the publication of this paper.

\section{Acknowledgments}

This work was supported by a Grant from the Chang Gung Memorial Hospital, Taiwan (CMRPG3B0761). HsingYu Chen and Yi-Hsuan Lin are coauthors.

\section{References}

[1] P. A. Cortesi, L. Scalone, A. Belisari et al., "Cost and quality of life in patients with severe chronic hand eczema refractory to standard therapy with topical potent corticosteroids," Contact Dermatitis, vol. 70, no. 3, pp. 158-168, 2014.

[2] R. J. Hay, N. E. Johns, H. C. Williams et al., “The global burden of skin disease in 2010: an analysis of the prevalence and impact of skin conditions," Journal of Investigative Dermatology, 2013.

[3] S. M. Langan, J. Schmitt, H. C. Williams, S. Smith, and K. S. Thomas, "How are eczema "flares" defined? A systematic review and recommendation for future studies," The British Journal of Dermatology, vol. 170, no. 3, pp. 548-556, 2014.

[4] S. V. Bershad, "In the clinic. Atopic dermatitis (eczema)," Annals of Internal Medicine, vol. 155, no. 9, p. ITC51-15, 2011, quiz ITC516.

[5] K. Eyerich and N. Novak, "Immunology of atopic eczema: overcoming the Th1/Th2 paradigm," Allergy, vol. 68, no. 8, pp. 974982, 2013.

[6] U. R. Hengge, T. Ruzicka, R. A. Schwartz, and M. J. Cork, "Adverse effects of topical glucocorticosteroids," Journal of the American Academy of Dermatology, vol. 54, no. 1, pp. 1-15, 2006.

[7] S. Moghadam-Kia and V. P. Werth, "Prevention and treatment of systemic glucocorticoid side effects," International Journal of Dermatology, vol. 49, no. 3, pp. 239-248, 2010.

[8] L. Schneider, S. Tilles, P. Lio et al., "Atopic dermatitis: a practice parameter update 2012," Journal of Allergy and Clinical Immunology, vol. 131, no. 2, pp. 295.e27-299.e27, 2013.

[9] L. C. Chang, N. Huang, Y. J. Chou, C. H. Lee, F. Y. Kao, and Y. T. Huang, "Utilization patterns of Chinese medicine and Western medicine under the National Health Insurance Program in Taiwan, a population-based study from 1997 to 2003," BMC Health Services Research, vol. 8, article 170, 2008.

[10] H. Y. Chen, Y. H. Lin, J. C. Wu et al., "Characteristics of pediatric traditional Chinese medicine users in Taiwan: a nationwide cohort study," Pediatrics, vol. 129, no. 6, pp. el485-e1492, 2012. 
[11] N. C. Armstrong and E. Ernst, "The treatment of eczema with Chinese herbs: a systematic review of randomized clinical trials," British Journal of Clinical Pharmacology, vol. 48, no. 2, pp. 262-264, 1999.

[12] V. Scheid, T. Ward, and V. Tuffrey, "Comparing TCM textbook descriptions of menopausal syndrome with the lived experience of London women at midlife and the implications for Chinese medicine research," Maturitas, vol. 66, no. 4, pp. 408-416, 2010.

[13] J. Hu, J. Zhang, W. Zhao, Y. Zhang, L. Zhang, and H. Shang, "Cochrane systematic reviews of Chinese herbal medicines: an overview," PLoS ONE, vol. 6, no. 12, Article ID e28696, 2011.

[14] S. Li and B. Zhang, "Traditional Chinese medicine network pharmacology: theory, methodology and application," Chinese Journal of Natural Medicines, vol. 11, no. 2, pp. 110-120, 2013.

[15] Y. H. Lin, Y. C. Chen, S. Hu, H. Y. Chen, J. L. Chen, and S. H. Yang, "Identifying core herbal treatments for urticaria using Taiwan's nationwide prescription database," Journal of Ethnopharmacology, vol. 148, no. 2, pp. 556-562, 2013.

[16] H. Y. Chen, Y. H. Lin, P. F. Thien et al., "Identifying core herbal treatments for children with asthma: implication from a Chinese Herbal medicine database in Taiwan," Evidence-Based Complementary and Alternative Medicine, vol. 2013, Article ID 125943, 10 pages, 2013.

[17] S. Wang, Y. Hu, W. Tan et al., "Compatibility art of traditional Chinese medicine: from the perspective of herb pairs," Journal of Ethnopharmacology, vol. 143, no. 2, pp. 412-423, 2012.

[18] S. Li, B. Zhang, D. Jiang, Y. Wei, and N. Zhang, "Herb network construction and co-module analysis for uncovering the combination rule of traditional Chinese herbal formulae," $B M C$ Bioinformatics, vol. 11, no. 11, article S6, 2010.

[19] G. Yuan, L. Zheng, W. Chong-Jun, F. Xin-Sheng, and X. JunYuan, "Chinese medicine formula network analysis for core herbal discovery," in Brain Informatics, F. Zanzotto, S. Tsumoto, N. Taatgen, and Y. Yao, Eds., pp. 255-264, Springer, Berlin, Germany, 2012.

[20] W. Tao, X. Xu, X. Wang et al., "Network pharmacology-based prediction of the active ingredients and potential targets of Chinese herbal Radix Curcumae formula for application to cardiovascular disease," Journal of Ethnopharmacology, vol. 145, no. 1, pp. 1-10, 2013.

[21] C. L. Cheng, Y. H. Y. Kao, S. J. Lin, C. H. Lee, and M. L. Lai, "Validation of the national health insurance research database with ischemic stroke cases in Taiwan," Pharmacoepidemiology and Drug Safety, vol. 20, no. 3, pp. 236-242, 2011.

[22] Y. C. Chen, H. Y. Yeh, J. C. Wu, I. Haschler, T. J. Chen, and T. Wetter, "Taiwan's national health insurance research database: administrative health care database as study object in bibliometrics," Scientometrics, vol. 86, no. 2, pp. 365-380, 2011.

[23] H. Y. Chen, Y. H. Lin, J. C. Wu et al., "Prescription patterns of Chinese herbal products for menopausal syndrome: analysis of a nationwide prescription database," Journal of Ethnopharmacology, vol. 137, no. 3, pp. 1261-1266, 2011.

[24] Y. M. Tai and H. W. Chiu, "Comorbidity study of ADHD: applying association rule mining (ARM) to National Health Insurance Database of Taiwan," International Journal of Medical Informatics, vol. 78, no. 12, pp. e75-e83, 2009.

[25] F. P. Chen, Y. Y. Kung, Y. C. Chen et al., "Frequency and pattern of Chinese herbal medicine prescriptions for chronic hepatitis in Taiwan," Journal of Ethnopharmacology, vol. 117, no. 1, pp. 8491, 2008.

[26] S. Yang, H. Chen, Y. Lin, and Y. Chen, "The exploration of disease pattern, zheng, for differentiation of allergic rhinitis in traditional Chinese medicine practice," Evidence-based Complementary and Alternative Medicine, vol. 2012, Article ID 521780, 7 pages, 2012.

[27] T. J. Chen, L. F. Chou, and S. J. Hwang, "Application of a data-mining technique to analyze coprescription patterns for antacids in taiwan," Clinical Therapeutics, vol. 25, no. 9, pp. 2453-2463, 2003.

[28] H. Y. Chen, Y. H. Lin, I. H. Su, Y. C. Chen, S. H. Yang, and J. L. Chen, "Investigation on chinese herbal medicine for primary dysmenorrhea: implication from a nationwide prescription database in taiwan," Complementary Therapies in Medicine, vol. 22, no. 1, pp. 116-125, 2014.

[29] D. H. Yang, J. H. Kang, Y. B. Park, Y. J. Park, H. S. Oh, and S. B. Kim, "Association rule mining and network analysis in oriental medicine," PLoS ONE, vol. 8, no. 3, Article ID e59241, 2013.

[30] K. Wakita and T. Tsurumi, "Finding community structure in mega-scale social networks: [extended abstract]," in Proceedings of the 16th International World Wide Web Conference (WWW '07), pp. 1275-1276, Banff, Canada, May 2007.

[31] N. Memon, J. J. Xu, D. L. Hicks, and H. Chen, Data Mining for Social Network Data, Springer, New York, NY, USA, 2010.

[32] H. Qu, Y. Zhang, X. Chai, and W. Sun, "Isoforsythiaside, an antioxidant and antibacterial phenylethanoid glycoside isolated from Forsythia suspensa," Bioorganic Chemistry, vol. 40, no. 1, pp. 87-91, 2012.

[33] S. J. Dai, Y. Ren, L. Shen, and D.-W. Zhang, "New alkaloids from Forsythia suspensa and their anti-inflammatory activities," Planta Medica, vol. 75, no. 4, pp. 375-377, 2009.

[34] Y. Hao, D. Li, and X. Piao, "Forsythia suspensa extract alleviates hypersensitivity induced by soybean $\beta$-conglycinin in weaned piglets," Journal of Ethnopharmacology, vol. 128, no. 2, pp. 412418, 2010.

[35] H. Qu, Y. Zhang, Y. Wang, B. Li, and W. Sun, "Antioxidant and antibacterial activity of two compounds (forsythiaside and forsythin) isolated from Forsythia suspensa," Journal of Pharmacy and Pharmacology, vol. 60, no. 2, pp. 261-266, 2008.

[36] H. J. Chen, Y. C. Lo, and W. Chiang, "Inhibitory effects of adlay bran (coix lachryma-jobi L. var. ma-yuen Stapf) on chemical mediator release and cytokine production in rat basophilic leukemia cells," Journal of Ethnopharmacology, vol. 141, no. 1, pp. 119-127, 2012.

[37] S. Shi, Y. Zhao, H. Zhou, Y. Zhang, X. Jiang, and K. Huang, "Identification of antioxidants from Taraxacum mongolicum by high-performance liquid chromatography-diode array detection-radical-scavenging detection-electrospray ionization mass spectrometry and nuclear magnetic resonance experiments," Journal of Chromatography A, vol. 1209, no. 1-2, pp. 145-152, 2008.

[38] S. Jiang, Y. Nakano, M. A. Rahman, R. Yatsuzuka, and C. Kamei, "Effects of a Dictamnus dasycarpus T. extract on allergic models in mice," Bioscience, Biotechnology and Biochemistry, vol. 72, no. 3, pp. 660-665, 2008.

[39] H. Kim, M. Kim, G. San Lee, W. G. An, and S. I. Cho, "Antiinflammatory activities of Dictamnus dasycarpus Turcz., root bark on allergic contact dermatitis induced by dinitrofluorobenzene in mice," Journal of Ethnopharmacology, vol. 149, no. 2, pp. 471-477, 2013.

[40] O. H. Kang, J. G. Choi, J. H. Lee, and D. Y. Kwon, "Luteolin isolated from the flowers of Lonicera japonica suppresses inflammatory mediator release by blocking NF- $\kappa$ B and MAPKs activation pathways in HMC-1 cells," Molecules, vol. 15, no. 1, pp. 385-398, 2010. 
[41] B. C. L. Chan, K. L.E. Hon, P. C. Leung et al., "Traditional Chinese medicine for atopic eczema: pentaHerbs formula suppresses inflammatory mediators release from mast cells," Journal of Ethnopharmacology, vol. 120, no. 1, pp. 85-91, 2008.

[42] T. Y. Wu, T. O. Khor, C. L. L. Saw et al., "Anti-inflammatory/antioxidative stress activities and differential regulation of Nrf2mediated genes by non-polar fractions of tea Chrysanthemum zawadskii and licorice Glycyrrhiza uralensis," The AAPS Journal, vol. 13, no. 1, pp. 1-13, 2011.

[43] J. H. Jin, T. M. Ngoc, K. Bae, Y. S. Kim, and H. P. Kim, "Inhibition of experimental atopic dermatitis by rhubarb (rhizomes of rheum tanguticum) and 5-lipoxygenase inhibition of its major constituent, emodin," Phytotherapy Research, vol. 25, no. 5, pp. 755-759, 2011.

[44] H. Matsuda, Y. Dai, Y. Ido, M. Yoshikawa, and M. Kubo, "Studies on Kochiae Fructus IV. Anti-allergic effects of $70 \%$ ethanol extract and its component, momordin Ic from dried fruits of Kochia scoparia L," Biological and Pharmaceutical Bulletin, vol. 20, no. 11, pp. 1165-1170, 1997.

[45] M. Nose, J. I. Sakushima, D. Harada, and Y. Ogihara, "Comparison of immunopharmacological actions of 8 kinds of kampo-hozais clinically used in atopic dermatitis on delayedtype hypersensitivity in mice," Biological and Pharmaceutical Bulletin, vol. 22, no. 1, pp. 48-54, 1999.

[46] K. Shichijo and H. Saito, "Effect of Chinese herbal medicines and disodium cromoglycate on IgE-dependent histamine release from mouse cultured mast cells," International Journal of Immunopharmacology, vol. 19, no. 11-12, pp. 677-682, 1998.

[47] H. Akamatsu, Y. Asada, and T. Horio, "Inhibitory effect of Shofu-san, a Japanese kampo medicine, on neutrophil functions in vitro," The American Journal of Chinese Medicine, vol. 26, no. 1, pp. 57-64, 1998.

[48] K. G. Xiu, K. Fuseda, T. Shibata, H. Tanaka, N. Inagaki, and H. Nagai, "Kampo medicines for mite antigen-induced allergic dermatitis in NC/Nga mice," Evidence-Based Complementary and Alternative Medicine, vol. 2, no. 2, pp. 191-199, 2005.

[49] Y. H. Wu, S. Y. Chuang, W. C. Hong, Y. J. Lai, Y. L. Chang, and J. H. S. Pang, "In vivo and in vitro inhibitory effects of a traditional Chinese formulation on LPS-stimulated leukocyteendothelial cell adhesion and VCAM-1 gene expression," Journal of Ethnopharmacology, vol. 140, no. 1, pp. 55-63, 2012.

[50] J. M. Hanifin and M. L. Reed, "A population-based survey of eczema prevalence in the United States," Dermatitis, vol. 18, no. 2, pp. 82-91, 2007.

[51] J. I. Silverberg and J. M. Hanifin, "Adult eczema prevalence and associations with asthma and other health and demographic factors: a US population-based study," Journal of Allergy and Clinical Immunology, vol. 132, no. 5, pp. 1132-1138, 2013.

[52] C. C. Jiang, Y. Tan, N. Zhao et al., "Analysis on regularity of TCM syndromes and chinese herbs application for eczema," Chinese Archives of Traditional Chinese Medicine, no. 11, pp. 2397-2399, 2013.

[53] I. H. Choi, S. Kim, Y. Kim, and Y. Yun, "The effect of TJ15 Plus TJ-17 on atopic dermatitis: a pilot study based on the principle of pattern identification," Journal of Alternative and Complementary Medicine, vol. 18, no. 6, pp. 576-582, 2012.

[54] J. Dai, S. Sun, J. Peng et al., "Exploration of macro-micro biomarkers for dampness-heat syndrome differentiation in different diseases," Evidence-Based Complementary and Alternative Medicine, vol. 2013, Article ID 706762, 9 pages, 2013.
[55] N. J. Mcnally, H. C. Williams, and D. R. Phillips, "Atopic eczema and the home environment," British Journal of Dermatology, vol. 145, no. 5, pp. 730-736, 2001.

[56] H. M. Cheng, L. C. Chiang, Y. M. Jan, G. W. Chen, and T. C. $\mathrm{Li}$, "The efficacy and safety of a Chinese herbal product (XiaoFeng-San) for the treatment of refractory atopic dermatitis: a randomized, double-blind, placebo-controlled trial," International Archives of Allergy and Immunology, vol. 155, no. 2, pp. 141-148, 2011.

[57] S. Jiang, Y. Nakano, M. A. Rahman, R. Yatsuzuka, and C. Kamei, "Effects of a Dictamnus dasycarpus T. extract on allergic models in mice," Bioscience, Biotechnology and Biochemistry, vol. 72, no. 3, pp. 660-665, 2008.

[58] H. Qu, Y. Zhang, X. Chai, and W. Sun, "Isoforsythiaside, an antioxidant and antibacterial phenylethanoid glycoside isolated from forsythia suspensa," Bioorganic Chemistry, vol. 40, no. 1, pp. 87-91, 2012.

[59] L. M. Lin, X. G. Zhang, J. J. Zhu, H. M. Gao, Z. M. Wang, and W. H. Wang, "Two new triterpenoid saponins from the flowers and buds of Lonicera japonica," Journal of Asian Natural Products Research, vol. 10, no. 10, pp. 925-929, 2008.

[60] Y. Hidaka, T. Kaneda, N. Amino, and K. Miyai, "Chinese medicine, Coix seeds increase peripheral cytotoxic T and NK cells," Biotherapy, vol. 5, no. 3, pp. 201-203, 1992.

[61] W. Zhao, J. L. Wolfender, K. Hostettmann, R. Xu, and G. Qin, "Antifungal alkaloids and limonoid derivatives from dictamnus dasycarpus," Phytochemistry, vol. 47, no. 1, pp. 7-11, 1998.

[62] Y. Wei, L. Shan, L. Qiao, R. Liu, Z. Hu, and W. Zhang, "Protective effects of Huang-Lian-Jie-Du-Tang against polymicrobial sepsis induced by cecal ligation and puncture in rats," Evidence-based Complementary and Alternative Medicine, vol. 2013, Article ID 909624, 11 pages, 2013.

[63] P. Y. Ong, T. Ohtake, C. Brandt et al., "Endogenous antimicrobial peptides and skin infections in atopic dermatitis," The New England Journal of Medicine, vol. 347, no. 15, pp. 1151-1160, 2002.

[64] J. T. Huang, M. Abrams, B. Tlougan, A. Rademaker, and A. S. Paller, "Treatment of staphylococcus aureus colonization in atopic dermatitis decreases disease severity," Pediatrics, vol. 123, no. 5, pp. e808-e814, 2009. 


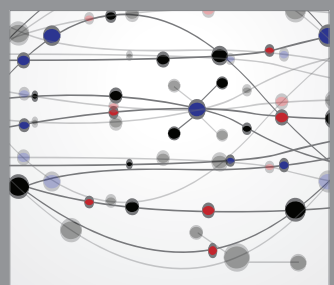

The Scientific World Journal
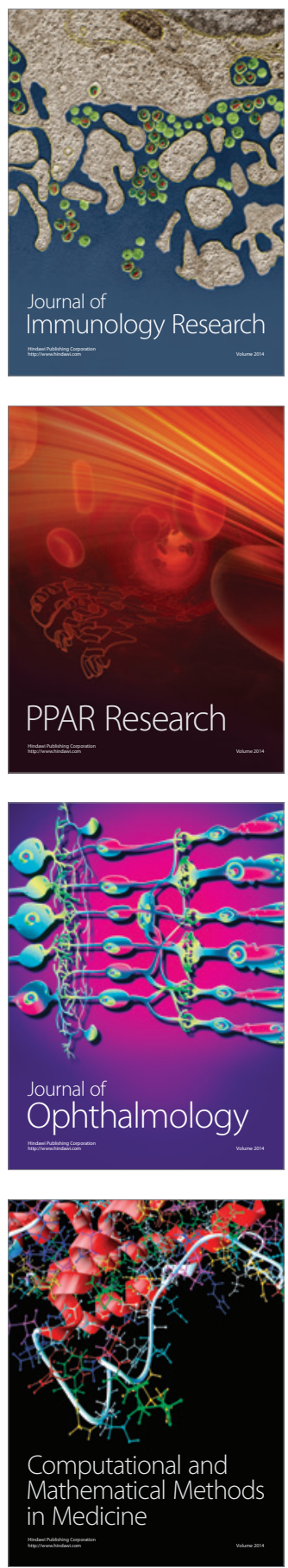

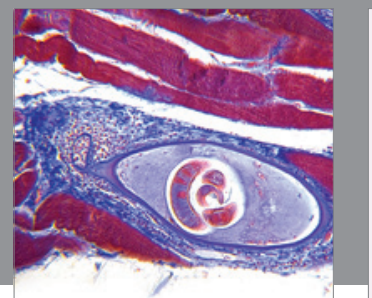

Gastroenterology

Research and Practice
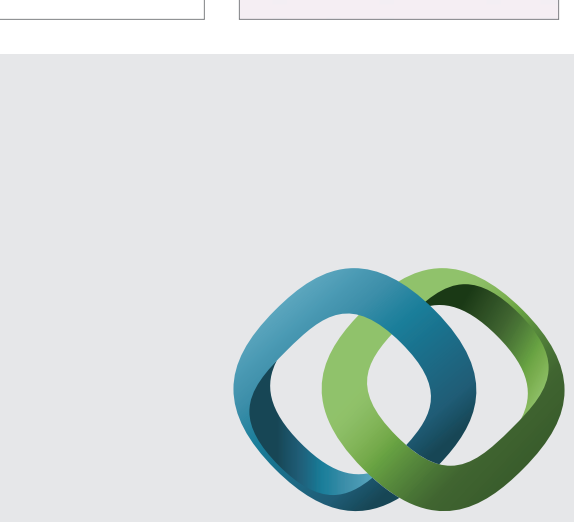

\section{Hindawi}

Submit your manuscripts at

http://www.hindawi.com
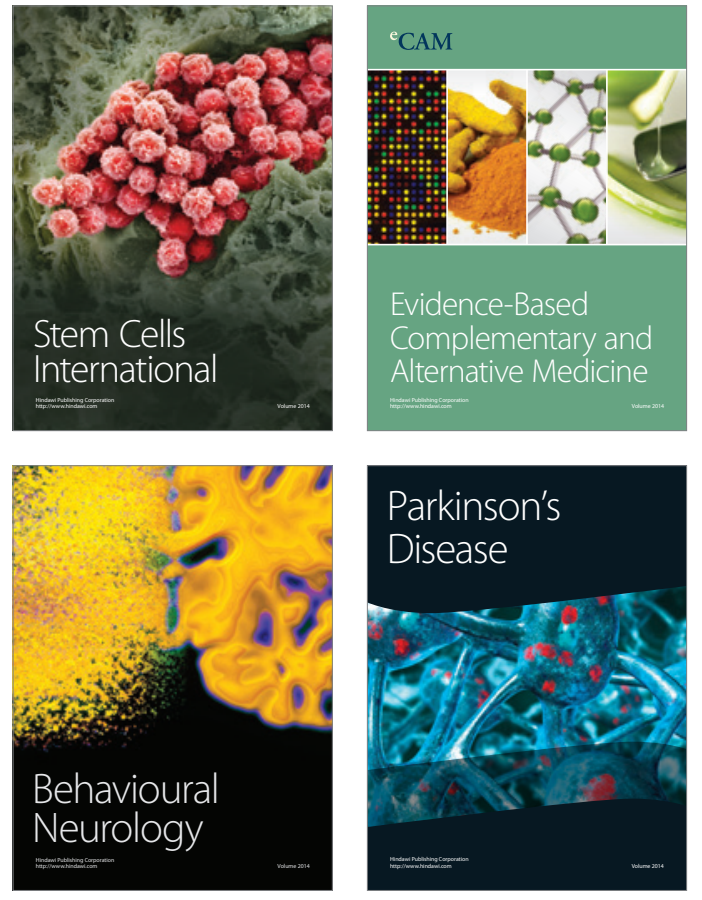
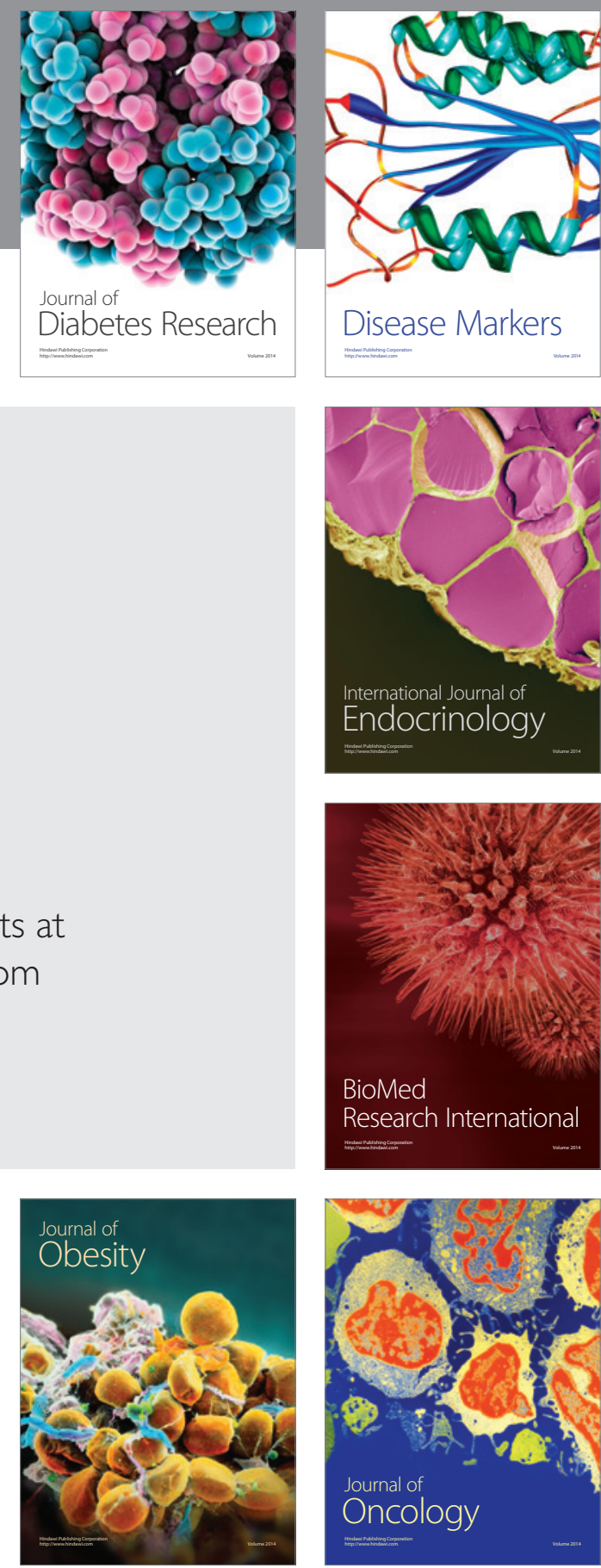

Disease Markers
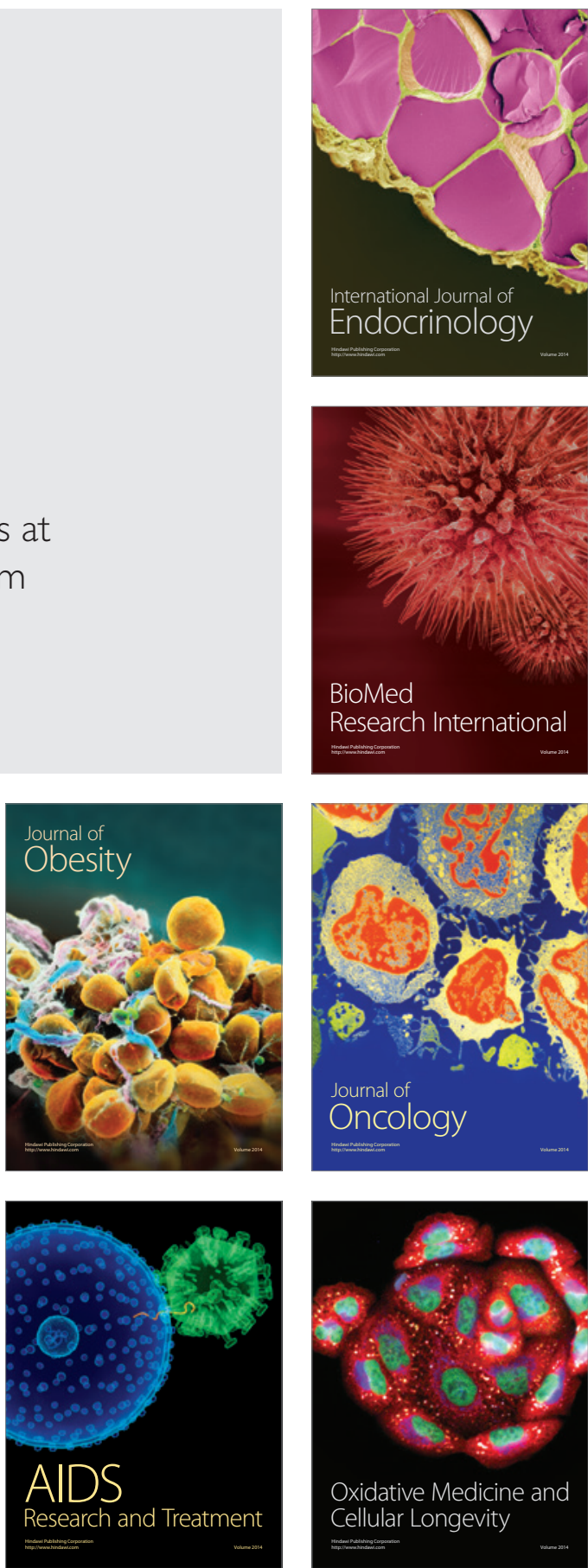